PROCEEDINGS OF THE

AMERICAN MATHEMATICAL SOCIETY

Volume 133, Number 11, Pages 3243-3253

S 0002-9939(05)08202-X

Article electronically published on June 20, 2005

\title{
COMPARISON THEOREMS OF HILLE-WINTNER TYPE FOR DYNAMIC EQUATIONS ON TIME SCALES
}

\author{
LYNN ERBE AND ALLAN PETERSON \\ (Communicated by Carmen C. Chicone)
}

\begin{abstract}
We obtain an analogue of the Hille-Wintner comparison theorem for the nonoscillation of second-order linear dynamic equations on time scales. Several examples are given including applications to difference equations.
\end{abstract}

\section{INTRODUCTION AND PRELIMINARY RESULTS}

We will be concerned with proving the analogue of the Hille-Wintner comparison theorem concerning the oscillation and nonoscillation of the two second-order linear dynamic equations

$$
\begin{aligned}
& L_{1} x=\left(r_{1}(t) x^{\Delta}\right)^{\Delta}+q_{1}(t) x^{\sigma}=0, \\
& L_{2} x=\left(r_{2}(t) x^{\Delta}\right)^{\Delta}+q_{2}(t) x^{\sigma}=0,
\end{aligned}
$$

where $r_{i}$ and $q_{i}, i=1,2$, are real-valued, right-dense continuous functions on a time scale $\mathbb{T} \subset \mathbb{R}$, with $\sup \mathbb{T}=\infty$. We also assume throughout that

$$
0<r_{1}(t) \leq r_{2}(t), \quad t \in \mathbb{T}, \quad \int_{a}^{\infty} \frac{1}{r_{1}(t)} \Delta t=\infty, \quad \int_{a}^{\infty} q_{i}(t) \Delta t
$$

exist for $i=1,2$, and that either

$$
0 \leq \int_{t}^{\infty} q_{2}(s) \Delta s \leq \int_{t}^{\infty} q_{1}(s) \Delta s
$$

for all large $t$, or

$$
\left|\int_{t}^{\infty} q_{2}(s) \Delta s\right| \leq \int_{t}^{\infty} q_{1}(s) \Delta s
$$

for all large $t$.

If $r_{i}>0, q_{i}, i=1,2$, are real-valued continuous functions satisfying

$$
0<r_{1}(t) \leq r_{2}(t), \quad q_{2}(t) \leq q_{1}(t)
$$

for $t$ in the real interval $[a, \infty)$, then the Sturm comparison theorem says that if

$$
\left(r_{1}(t) x^{\prime}\right)^{\prime}+q_{1}(t) x=0
$$

Received by the editors May 21, 2004.

2000 Mathematics Subject Classification. Primary 39A10.

Key words and phrases. Comparison theorems, linear oscillation, Hille-Wintner, time scale. 
is nonoscillatory on $[a, \infty)$ (i.e. all nontrivial solutions have finitely many zeros in $[a, \infty))$, then

$$
\left(r_{2}(t) x^{\prime}\right)^{\prime}+q_{2}(t) x=0
$$

is also nonoscillatory on $[a, \infty)$. The Hille-Wintner theorem replaces the point-wise comparisons on the coefficient functions by integral comparisons. (See Remark 2.2 for additional details.) There are many recent additional results for oscillation of dynamic equations on time scales for both the linear and nonlinear case (see, e.g., [1], 4]-[13, 18], 20]).

For completeness, we recall the following concepts related to the notion of time scales. A time scale $\mathbb{T}$ is an arbitrary nonempty closed subset of the real numbers $\mathbb{R}$ and, since oscillation of solutions is our primary concern, we make the blanket assumption that $\sup \mathbb{T}=\infty$. We assume throughout that $\mathbb{T}$ has the topology that it inherits from the standard topology on the real numbers $\mathbb{R}$. The forward jump operator and the backward jump operator are defined by

$$
\sigma(t):=\inf \{s \in \mathbb{T}: s>t\}, \quad \rho(t):=\sup \{s \in \mathbb{T}: s<t\},
$$

where $\sup \emptyset=\inf \mathbb{T}$. A point $t \in \mathbb{T}$ is said to be left-dense if $\rho(t)=t$ and $t>\inf \mathbb{T}$, right-dense if $\sigma(t)=t$, left-scattered if $\rho(t)<t$ and right-scattered if $\sigma(t)>t$. A function $g: \mathbb{T} \rightarrow \mathbb{R}$ is said to be right-dense continuous (rdcontinuous) provided $g$ is continuous at right-dense points and at left-dense points in $\mathbb{T}$, left-hand limits exist and are finite. The set of all such rd-continuous functions is denoted by $C_{r d}(\mathbb{T})$. The graininess function $\mu$ for a time scale $\mathbb{T}$ is defined by $\mu(t):=\sigma(t)-t$, and for any function $f: \mathbb{T} \rightarrow \mathbb{R}$ the notation $f^{\sigma}(t)$ denotes $f(\sigma(t))$.

Definition 1.1. Fix $t \in \mathbb{T}$ and let $x: \mathbb{T} \rightarrow \mathbb{R}$. Define $x^{\Delta}(t)$ to be the number (if it exists) with the property that given any $\epsilon>0$ there is a neighbourhood $U$ of $t$ with

$$
\left|[x(\sigma(t))-x(s)]-x^{\Delta}(t)[\sigma(t)-s]\right| \leq \epsilon|\sigma(t)-s|, \quad \text { for all } s \in U .
$$

In this case, we say $x^{\Delta}(t)$ is the (delta) derivative of $x$ at $t$ and that $x$ is (delta) differentiable at $t$.

The following theorem is due to Hilger [15].

Theorem 1.2. Assume that $g: \mathbb{T} \rightarrow \mathbb{R}$ and let $t \in \mathbb{T}$.

(i) If $g$ is differentiable at $t$, then $g$ is continuous at $t$.

(ii) If $g$ is continuous at $t$ and $t$ is right-scattered, then $g$ is differentiable at $t$ with

$$
g^{\Delta}(t)=\frac{g(\sigma(t))-g(t)}{\sigma(t)-t} .
$$

(iii) If $g$ is differentiable and $t$ is right-dense, then

$$
g^{\Delta}(t)=\lim _{s \rightarrow t} \frac{g(t)-g(s)}{t-s} .
$$

(iv) If $g$ is differentiable at $t$, then $g(\sigma(t))=g(t)+\mu(t) g^{\Delta}(t)$.

Definition 1.3. If $G^{\Delta}(t)=g(t)$, then the Cauchy (delta) integral of $g$ is defined by

$$
\int_{a}^{t} g(s) \Delta s:=G(t)-G(a)
$$


For a more general definition of the delta integral see [2], 3].

We assume throughout that $t_{0} \geq 0$ and $t_{0} \in \mathbb{T}$. By the interval $\left[t_{0}, \infty\right)$ we mean the set $\left[t_{0}, \infty\right) \cap \mathbb{T}$. The theory of time scales dates back to Hilger [15]. The monographs [2], 3] and [17] also provide an excellent introduction.

The following lemma [12, Lemma 13] will be useful in the proof of Theorem 2.1

Lemma 1.4. Assume

$$
\liminf _{t \rightarrow \infty} \int_{T}^{t} q_{1}(s) \Delta s \geq 0 \quad \text { and not identically } 0
$$

for all large $T$, and $\int_{a}^{\infty} \frac{1}{r_{1}(s)} \Delta s=\infty$. If (1.1) has a positive solution $x=x(t)$ for all sufficiently large $t$, then $x^{\Delta}(t)>0$ for all sufficiently large $t$.

\section{MAIN RESUltS}

We recall that a solution of equation (1.1) is said to be oscillatory on $[a, \infty)$ in case it is neither eventually positive nor eventually negative. Otherwise, the solution is said to be nonoscillatory on $[a, \infty)$. Equation (1.1) is said to be oscillatory on $[a, \infty)$ in case all of its solutions are oscillatory on $[a, \infty)$.

We now state and prove our main result.

Theorem 2.1 (Hille-Wintner Theorem). Assume that (1.3) and (1.4) hold. Let

$$
\hat{\mathbb{T}}:=\{t \in \mathbb{T}: \mu(t)>0\}
$$

and let $\chi$ denote the characteristic function of $\hat{\mathbb{T}}$. Assume further that there is an $M>0$ such that

$$
r_{1}(t) \chi(t) \leq M \mu(t), \quad t \in \mathbb{T}
$$

Then if $L_{1} x=0$ is nonoscillatory on $[a, \infty)$, it follows that $L_{2} x=0$ is nonoscillatory on $[a, \infty)$.

Proof. We can assume without loss of generality that $\sup \hat{\mathbb{T}}=\infty$, since otherwise $\mathbb{T}$ is eventually a real interval, (2.1) holds trivially, and so the result reduces to the usual Hille-Wintner theorem. Since $L_{1} x=0$ is nonoscillatory on $[a, \infty)$, there is a $T \in[a, \infty)$ and a solution $x$ of (1.1) with $x(t)>0$ on $[T, \infty)$. If we make the Riccati substitution

$$
z(t):=\frac{r_{1}(t) x^{\Delta}(t)}{x(t)}, \quad t \geq T
$$

then (cf. [2, Theorem 4.36]) $z$ is a solution of the Riccati equation

$$
R_{1} z=z^{\Delta}+q_{1}(t)+\frac{z^{2}}{r_{1}(t)+\mu(t) z}=0
$$

on $[T, \infty)$ and satisfies

$$
r_{1}(t)+\mu(t) z(t)>0
$$


on $[T, \infty)$. Now let us denote

$$
F(t):=\frac{z^{2}(t)}{r_{1}(t)+\mu(t) z(t)} \geq 0 .
$$

Then integrating (2.2) gives

$$
z(t)+\int_{T}^{t} q_{1}(s) \Delta s+\int_{T}^{t} F(s) \Delta s=z(T) .
$$

If $q_{1}(t) \equiv 0$ for large $t$, then (1.4) implies $q_{2}(t) \equiv 0$ for large $t$, and the conclusion of the theorem is immediate. Hence we can assume that $q_{1}(t) \not \equiv 0$ for large $t$ and in this case (1.4) implies (1.6). Using (1.3) it follows without loss of generality from Lemma 1.4 that $z(t)>0$ for $t \geq T$ and by (1.4), $\int_{T}^{t} q_{1}(s) \Delta s \geq 0$ for $t \geq T$, where $T$ is sufficiently large. Then an easy application of (1.3) gives that $\int_{T}^{\infty} F(s) \Delta s<$ $\infty$. Again using (1.3) we get from (2.4) that $\lim _{t \rightarrow \infty} z(t)$ exists. We claim that $\lim _{t \rightarrow \infty} z(t)=0$. Let $\left\{t_{k}\right\} \subset \hat{\mathbb{T}}$ with $\lim _{k \rightarrow \infty} t_{k}=\infty$. By (2.4) we have that

$$
0 \leq \int_{T}^{t_{k}} q_{1}(s) \Delta s+\int_{T}^{t_{k}} F(s) \Delta s \leq z(T) .
$$

We recall (cf. 22 Theorem 1.75]) that $\int_{t}^{\sigma(t)} g(s) \Delta s=\mu(t) g(t)$ and so we have, for $n_{0}$ sufficiently large,

$$
\sum_{k=n_{0}}^{\infty} \mu\left(t_{k}\right) F\left(t_{k}\right)=\sum_{k=n_{0}}^{\infty} \int_{t_{k}}^{\sigma\left(t_{k}\right)} F(t) \Delta t \leq \int_{T}^{\infty} F(s) \Delta s<\infty .
$$

Therefore it follows that

$$
0=\lim _{k \rightarrow \infty} \mu\left(t_{k}\right) F\left(t_{k}\right)=\lim _{k \rightarrow \infty} \frac{\mu\left(t_{k}\right) z^{2}\left(t_{k}\right)}{r_{1}(t)+\mu\left(t_{k}\right) z\left(t_{k}\right)}=\lim _{k \rightarrow \infty} \frac{z^{2}\left(t_{k}\right)}{\frac{r_{1}(t)}{\mu\left(t_{k}\right)}+z\left(t_{k}\right)} .
$$

Hence given an $\varepsilon>0$ there is a positive integer $k_{0}$ such that

$$
0<\frac{z^{2}\left(t_{k}\right)}{\frac{r_{1}\left(t_{k}\right)}{\mu\left(t_{k}\right)}+z\left(t_{k}\right)}<\varepsilon
$$

for $k \geq k_{0}$. This implies that

$$
z^{2}\left(t_{k}\right)<\varepsilon\left(\frac{r_{1}\left(t_{k}\right)}{\mu\left(t_{k}\right)}+z\left(t_{k}\right)\right),
$$

which implies that

$$
\left(z\left(t_{k}\right)-\frac{\varepsilon}{2}\right)^{2}<\frac{\varepsilon^{2}}{4}+\varepsilon \frac{r_{1}\left(t_{k}\right)}{\mu\left(t_{k}\right)} \leq \frac{\varepsilon^{2}}{4}+\varepsilon M .
$$

Therefore

$$
\left|z\left(t_{k}\right)-\frac{\varepsilon}{2}\right|<\frac{\varepsilon}{2}+\sqrt{\varepsilon M}
$$

and consequently

$$
\left|z\left(t_{k}\right)\right|<\varepsilon+\sqrt{\varepsilon M} .
$$

Since $\varepsilon>0$ is arbitrary, we get that

$$
\lim _{k \rightarrow \infty} z\left(t_{k}\right)=0 .
$$


But from (2.4) we know that $\lim _{t \rightarrow \infty} z(t)=z_{0} \geq 0$ exists, and hence we get the desired result

$$
\lim _{t \rightarrow \infty} z(t)=0 .
$$

Now letting $t \rightarrow \infty$ in (2.4) we get

Define, for large $t$,

$$
\int_{T}^{\infty} q_{1}(s) \Delta s+\int_{T}^{\infty} F(s) \Delta s=z(T) .
$$

Then using (1.4),

$$
v(t):=\int_{t}^{\infty} q_{2}(s) \Delta s+\int_{t}^{\infty} F(s) \Delta s
$$

$$
0 \leq v(t) \leq \int_{t}^{\infty} q_{1}(s) \Delta s+\int_{t}^{\infty} F(s) \Delta s=z(t) .
$$

Note that

$$
v^{\Delta}(t)=-q_{2}(t)-F(t)=-q_{2}(t)-\frac{z^{2}(t)}{r_{1}(t)+\mu(t) z(t)} .
$$

We now claim that

$$
\frac{z^{2}(t)}{r_{1}(t)+\mu(t) z(t)} \geq \frac{v^{2}(t)}{r_{1}(t)+\mu(t) v(t)}, \quad t \geq T .
$$

This follows from the fact that for each fixed $t, H(w):=\frac{w^{2}}{r_{1}(t)+\mu(t) w}$ is strictly increasing for $w \geq 0$ and the fact that $v(t) \geq 0$. But this implies that the Riccati dynamic inequality

$$
v^{\Delta}+q_{2}(t)+\frac{v^{2}}{r_{1}(t)+\mu(t) v} \leq 0
$$

has a solution on $[T, \infty)$ with $r_{1}(t)+\mu(t) v(t)>0$, and this means (cf. [12, Lemma 5]) that

$$
\left(r_{1}(t) x^{\Delta}\right)^{\Delta}+q_{2}(t) x^{\sigma}=0
$$

nonoscillatory on $[a, \infty)$. Since $0<r_{1}(t) \leq r_{2}(t)$ we have by the Sturm comparison theorem [2, Theorem 5.60] that $L_{2} x=0$ is nonoscillatory on $[a, \infty)$.

Remark 2.2. If $\mathbb{T}=\mathbb{R}, r_{i}(t) \equiv 1, i=1,2$, and (1.4) holds, then the above result was first obtained by Hille [16] with the additional assumption that the $q_{i}(t), i=1,2$, are positive. Wintner in [21] showed that (1.4) is sufficient for the conclusion to hold without the assumption of positivity. Taam [20] showed later that the conclusion of the theorem holds with (1.5) replacing (1.4). We shall see, however, that for the general case of time scales, additional assumptions on $q_{1}(t)$ and on the set of right-scattered points are needed to obtain the analogous result when (1.5) replaces (1.4).

Theorem 2.3. Suppose that (1.3) and (1.5) hold and, in addition, assume there exists $0<m<M$ such that

$$
m \mu(t) \leq r_{1}(t) \chi(t) \leq M \mu(t), \quad t \in \mathbb{T} .
$$

Suppose also that $q_{1}(t)$ is positive near $\infty$ for $t \in \hat{\mathbb{T}}$ and

$$
\liminf _{t \rightarrow \infty, t \in \mathbb{T}} \frac{q_{2}(t)}{q_{1}(t)}>-1 .
$$

Then $L_{1} x=0$ nonoscillatory on $[a, \infty)$ implies $L_{2} x=0$ is nonoscillatory on $[a, \infty)$. 
Proof. Let $x(t), z(t)$, and $F(t)$ be as in the proof of Theorem 2.1. Then

$$
z(t)=\int_{t}^{\infty} q_{1}(s) \Delta s+\int_{t}^{\infty} F(s) \Delta s
$$

and $z(t)$ satisfies (2.3) for $t \in[T, \infty)$. Define

$$
v(t):=\int_{t}^{\infty} q_{2}(s) \Delta s+\int_{t}^{\infty} F(s) \Delta s .
$$

Note that, for $t \in[T, \infty)$,

$$
\begin{aligned}
|v(t)| & \leq\left|\int_{t}^{\infty} q_{2}(s) \Delta s\right|+\int_{t}^{\infty} F(s) \Delta s \\
& \leq \int_{t}^{\infty} q_{1}(s) \Delta s+\int_{t}^{\infty} F(s) \Delta s \\
& =z(t) .
\end{aligned}
$$

We would like to prove that

$$
\frac{z^{2}(t)}{r_{1}(t)+\mu(t) z(t)} \geq \frac{v^{2}(t)}{r_{1}(t)+\mu(t) v(t)},
$$

for large $t$. Note that for any right-dense point and for any point where $z(t)=v(t)$, (2.8) is true. Hence it remains to show that (2.8) is true for all sufficiently large right-scattered points where $z(t)>v(t)$. For such points note that (2.8) is equivalent to

$$
r_{1}(t)(z(t)+v(t)) \geq-\mu(t) v(t) z(t) .
$$

Evidently, $z(t)+v(t)>0$, and so (2.9) is equivalent to

$$
\frac{r_{1}(t)}{\mu(t)} \geq-\frac{v(t) z(t)}{z(t)+v(t)}=-\frac{v(t)}{1+\frac{v(t)}{z(t)}} .
$$

If there is no sequence $\left\{t_{n}\right\}$ of points in $\hat{\mathbb{T}}$ such that $\lim _{n \rightarrow \infty} t_{n}=\infty$ and

$$
\liminf _{n \rightarrow \infty} \frac{v\left(t_{n}\right)}{z\left(t_{n}\right)}=-1
$$

then, since $\lim _{t \rightarrow \infty} v(t)=0$, it would follow that

$$
\lim _{t \rightarrow \infty, t \in \hat{\mathbb{T}}} \frac{v(t)}{1+\frac{v(t)}{z(t)}}=0 .
$$

Since for $t \in \hat{\mathbb{T}}$, we have

$$
\frac{r_{1}(t)}{\mu(t)} \geq m>0,
$$

it would follow that (2.10) and hence (2.8) hold for all sufficiently large $t$. It remains to prove that (2.11) does not hold for any sequence in $\hat{\mathbb{T}}$ tending to $\infty$. We do this by contradiction. So assume there is such a sequence $\left\{t_{n}\right\}$ with

$$
\lim _{n \rightarrow \infty} \frac{v\left(t_{n}\right)}{z\left(t_{n}\right)}=-1 .
$$

Since we are assuming that $q_{1}(t)>0$ for large $t$ we get that

$$
z^{\Delta}(t)=-q_{1}(t)-\frac{z^{2}(t)}{r_{1}(t)+\mu(t) z(t)}<0
$$


for large $t$. Since $z(t)>0$ and $z^{\Delta}(t)<0$ for large $t$ we can apply L'Hôpital's rule for the time scale case [2, Theorem 1.120] to get

$$
\begin{aligned}
-1 & =\lim _{n \rightarrow \infty} \frac{v\left(t_{n}\right)}{z\left(t_{n}\right)}=\lim _{n \rightarrow \infty} \frac{v^{\Delta}\left(t_{n}\right)}{z^{\Delta}\left(t_{n}\right)} \\
& =\lim _{n \rightarrow \infty} \frac{-q_{2}\left(t_{n}\right)-\frac{z^{2}\left(t_{n}\right)}{r_{1}\left(t_{n}\right)+\mu\left(t_{n}\right) z\left(t_{n}\right)}}{z_{1}\left(t_{n}\right)-\frac{z^{2}\left(t_{n}\right)}{r_{1}\left(t_{n}\right)+\mu\left(t_{n}\right) z\left(t_{n}\right)}} \\
& =\lim _{n \rightarrow \infty} \frac{\left(r_{1}\left(t_{n}\right)+\mu\left(t_{n}\right) z\left(t_{n}\right)\right) q_{2}\left(t_{n}\right)+z^{2}\left(t_{n}\right)}{\left(r_{1}\left(t_{n}\right)+\mu\left(t_{n}\right) z\left(t_{n}\right)\right) q_{1}\left(t_{n}\right)+z^{2}\left(t_{n}\right)} .
\end{aligned}
$$

That is,

$$
\lim _{n \rightarrow \infty} \frac{a_{n} \frac{q_{2}\left(t_{n}\right)}{q_{1}\left(t_{n}\right)}+b_{n}}{a_{n}+b_{n}}=-1
$$

where

$$
a_{n}:=r_{1}\left(t_{n}\right)+\mu\left(t_{n}\right) z\left(t_{n}\right)>0, \quad b_{n}:=\frac{z^{2}\left(t_{n}\right)}{q_{1}\left(t_{n}\right)}>0 .
$$

By (2.7), there is an $\varepsilon>0$ such that

$$
\frac{q_{2}\left(t_{n}\right)}{q_{1}\left(t_{n}\right)} \geq-1+\varepsilon
$$

for large $n$, where we suppose also that $\varepsilon<2$. Let $0<\delta<\varepsilon$ be given. Then by (2.13) for large $n$,

$$
a_{n} \frac{q_{2}\left(t_{n}\right)}{q_{1}\left(t_{n}\right)}+b_{n}<(-1+\delta)\left(a_{n}+b_{n}\right) .
$$

Hence we have for large $n$ that

$$
(-1+\varepsilon) a_{n}+b_{n} \leq a_{n} \frac{q_{2}\left(t_{n}\right)}{q_{1}\left(t_{n}\right)}+b_{n}<(-1+\delta)\left(a_{n}+b_{n}\right) .
$$

But this implies that

$$
(\varepsilon-\delta) a_{n}<(-2+\delta) b_{n}<0
$$

for large $n$, which is a contradiction, since the left-hand side is positive. Therefore it follows that (2.8) holds and this implies that

$$
v^{\Delta}(t) \leq-q_{2}(t)-\frac{v^{2}(t)}{r_{1}(t)+\mu(t) v(t)} .
$$

Consequently, it follows that $\left(r_{1}(t) x^{\Delta}\right)^{\Delta}+q_{2}(t) x^{\sigma}$ is nonoscillatory on $[a, \infty)$. Then by the Sturm comparison theorem $L_{2} x=0$ is nonoscillatory on $[a, \infty)$.

\section{EXAMPLES}

Example 3.1. Consider the Euler-Cauchy-like equation

$$
x^{\Delta \Delta}+\frac{\gamma}{t \sigma(t)} x^{\sigma}=0 .
$$


(This dynamic equation in general is not an Euler-Cauchy equation according to the definition given in [2]; however if $\mathbb{T}=\mathbb{R}$, then this is an Euler-Cauchy differential equation.) For $\gamma<\frac{1}{4}$ it is known [18] that [3.1] is nonoscillatory near infinity provided

$$
\mu(t)=o(t) \quad \text { as } t \rightarrow \infty, \quad \text { i.e. } \quad \frac{\mu(t)}{t} \rightarrow 0 \quad \text { as } t \rightarrow \infty \text {. }
$$

Since $\int_{t}^{\infty} \frac{\gamma}{s \sigma(s)} \Delta s=\frac{\gamma}{t}$, we get from Theorem 2.1 that if (3.2) holds, if $\mu(t)$ is bounded below by a positive number for $t \in \hat{\mathbb{T}}$, and if

$$
0 \leq \limsup _{t \rightarrow \infty} t \int_{t}^{\infty} q(s) \Delta s<\frac{1}{4}
$$

then $x^{\Delta \Delta}+q(t) x^{\sigma}=0$ is nonoscillatory on $[a, \infty)$.

To see that the above result is sharp we note the following example.

Example 3.2. If

$$
\liminf _{t \rightarrow \infty} t \int_{t}^{\infty} q(s) \Delta s>\frac{1}{4}
$$

then $x^{\Delta \Delta}+q(t) x^{\sigma}=0$ is oscillatory on $[a, \infty)$. We show that this follows from the following result, which is the linear version of [13. Corollary 3.3].

Theorem 3.3. Assume that $q(t)$ is positive, $\int_{a}^{\infty} \frac{1}{r(t)} \Delta t=\infty$ holds and for any $t_{0} \geq a$ there is a $t_{1}>t_{0}$ such that

$$
\limsup _{t \rightarrow \infty} \int_{t_{1}}^{t}\left[\sigma(s)\left[q(s)-\left(\frac{1}{2 s \sigma(s)}\right)+\frac{1}{4 s^{2} C_{1}(s)}\right]-\frac{A_{1}^{2}(s) C_{1}(s)}{4 B_{1}(s)}\right] \Delta s=\infty
$$

where

$$
\begin{aligned}
& A_{1}(s)=\frac{-1}{s C_{1}(s)}\left(1+\frac{1}{s} \mu(s)-C_{1}(s)\right), \\
& B_{1}(s)=\frac{s+\mu(s)}{s^{2}}, \quad C_{1}(s)=1+\frac{\mu(s)}{\left(s-t_{0}\right)} .
\end{aligned}
$$

Then $\left(r(t) x^{\Delta}\right)^{\Delta}+q(t) x^{\sigma}=0$ is oscillatory on $[a, \infty)$.

An easy calculation shows that for the dynamic equation (3.1) we have

$$
C_{1}(s)=\frac{\sigma(s)-t_{0}}{s-t_{0}}, \quad A_{1}(s)=-\frac{\sigma(s)}{s^{2} C_{1}(s)}+\frac{1}{s}, \quad B_{1}(s)=\frac{\sigma(s)}{s^{2}} .
$$

One can then show that

$$
\frac{\sigma(s)}{4 s^{2} C_{1}(s)}-\frac{A_{1}^{2}(s) C_{1}(s)}{4 B_{1}(s)}=\frac{1}{4 s}\left(2-\frac{s\left(\sigma(s)-t_{0}\right)}{\sigma(s)\left(s-t_{0}\right)}\right) .
$$


Since this last expression is asymptotic to $\frac{1}{4 s}$ as $s \rightarrow \infty$ and since $\int_{t_{1}}^{\infty} \frac{1}{s} \Delta s=\infty$, for any time scale which is unbounded above, we get that for $t_{1}>t_{0}$,

$$
\limsup _{t \rightarrow \infty} \int_{t_{1}}^{t}\left(\frac{\gamma}{s}-\frac{1}{2 s}+\frac{\sigma(s)}{4 s^{2} C_{1}(s)}-\frac{A_{1}^{2}(s) C_{1}(s)}{4 b_{1}(s)}\right) \Delta s=\infty
$$

if $\gamma>\frac{1}{4}$. Hence by Theorem 3.3 we have that if $\gamma>\frac{1}{4}$, then (3.1) is oscillatory on $[a, \infty)$.

In Example 3.1 we assumed that $\mathbb{T}$ was a time scale satisfying (3.2). A time scale that is important in the theory of orthogonal polynomials and quantum theory (cf., e.g., [14, [19]) is $\mathbb{T}=q^{\mathbb{N}_{0}}:=\left\{1, q, q^{2}, q^{3}, \cdots\right\}$. For this time scale, $\mu(t)=(q-1) t$, so (3.2) is not satisfied. We now give an application of Theorem 2.1 for this time scale.

Example 3.4. It is not difficult to show that $x(t)=t^{\alpha}$ is a solution of the dynamic equation

$$
x^{\Delta \Delta}+\frac{C_{\alpha}}{t \sigma(t)} x^{\sigma}=0,
$$

where $C_{\alpha}:=\frac{\left(q^{\alpha}-1\right)\left(q^{1-\alpha}-1\right)}{(q-1)^{2}}$, for $t \in \mathbb{T}=q^{\mathbb{N}_{0}}, q>1$. If we let $0<\alpha<1$, then $C_{\alpha}>0$. We have with $t=q^{n}$,

$$
\int_{t}^{\infty} \frac{C_{\alpha}}{s \sigma(s)} \Delta s=\frac{C_{\alpha}}{t}=\frac{\left(q^{\alpha}-1\right)\left(q^{1-\alpha}-1\right)}{(q-1)^{2} q^{n}}
$$

and so if $Q(t)$ is defined on $\mathbb{T}$, then

$$
\int_{t}^{\infty} Q(s) \Delta s=(q-1) \sum_{k=n}^{\infty} q^{k} Q\left(q^{k}\right) .
$$

Hence, if

$$
0 \leq \sum_{k=n}^{\infty} q^{k} Q\left(q^{k}\right) \leq \frac{\left(q^{\alpha}-1\right)\left(q^{1-\alpha}-1\right)}{(q-1)^{3} q^{n}}
$$

for all large $n$, then by Theorem 2.1, $x^{\Delta \Delta}+Q(t) x^{\sigma}=0$ is nonoscillatory on $\mathbb{T}=q^{\mathbb{N}_{0}}$.

Remark 3.5. It was shown in 9, Example 16] (see also 2, Example 4.48]) that $x^{\Delta \Delta}+\frac{c}{(q-1) t \sigma(t)} x^{\sigma}=0$ is oscillatory on $\mathbb{T}=q^{\mathbb{N}_{0}}, q>1$, if $c>1$. We can use the result of Example 3.4 to show that this is sharp. To see this, notice that for fixed $0<\alpha<1, h(x):=\frac{\left(x^{\alpha}-1\right)\left(x^{1-\alpha}-1\right)}{x-1}$ satisfies $h^{\prime}(x)>0, x>1$ and $\lim _{x \rightarrow \infty} h(x)=1$. Hence given any $c_{0}<1$, we can choose $q$ sufficiently large so that $h(q)=(q-1) C_{\alpha}>$ $c_{0}$ and so since (3.4) has the nonoscillatory solution $x(t)=t^{\alpha}$ on $\mathbb{T}=q^{\mathbb{N}}$, it follows by the Sturm comparison theorem that $x^{\Delta \Delta}+\frac{c_{0}}{(q-1) t \sigma(t)} x^{\sigma}=0$ is nonoscillatory on $\mathbb{T}=q^{\mathbb{N}_{0}}$.

Example 3.6. We note that Examples 3.1 and 3.4 involve an application of Theorem 2.1 and that Theorem 2.3 does not apply since (2.6), 2.7) need not hold (i.e., $\mu(t)$ is unbounded in Example 3.4. As a simple example of Theorem 2.3, let

$$
r_{i}(t) \equiv 1, i=1,2, \quad q_{1}(t):=\frac{\gamma}{t \sigma(t)}, \quad q_{2}(t):=\frac{\lambda}{t^{2}}(-1)^{t}
$$


for $t \in \mathbb{T}=\mathbb{N}:=\{1,2,3, \cdots\}$, where $\lambda>0$. We have that

$$
\int_{t}^{\infty} q_{1}(s) \Delta s=\frac{\gamma}{t} \quad \text { and } \quad\left|\int_{t}^{\infty} q_{2}(s) \Delta s\right| \leq \frac{\lambda}{t^{2}}
$$

for $t \in \mathbb{N}$. Notice that $\int_{t}^{\infty} q_{2}(s) \Delta s$ assumes positive and negative values for arbitrarily large $t$, so that Theorem 2.1 does not apply. Clearly, for any $\lambda, \gamma>0$, (1.5) holds for all large $t$. Moreover

$$
\frac{q_{2}(t)}{q_{1}(t)}=\frac{\lambda}{\gamma}(-1)^{t}\left(1+\frac{1}{t}\right)
$$

so that (2.7) holds if $\lambda<\gamma$. Hence, if $0<\lambda<\gamma<\frac{1}{4}$, then $L_{2} x=0$ is nonoscillatory on $\mathbb{N}$ by Theorem 2.3 We note that the results in the above examples may not be established by any other criteria known to the authors. More sophisticated examples are left to the reader.

\section{REFERENCES}

[1] E. Akın-Bohner, M. Bohner, and S.H. Saker, Oscillation criteria for a certain class of second order Emden-Fowler dynamic equations, Electron. Trans. Numer. Anal., to appear.

[2] M. Bohner and A. Peterson, Dynamic Equations on Time Scales: An Introduction with Applications, Birkhäuser, Boston, 2001. MR1843232 (2002c:34002)

[3] M. Bohner and A. Peterson, Advances in Dynamic Equations on Time Scales, Birkhäuser, Boston, 2003. MR1962542 (2004d:34003)

[4] M. Bohner and S. H. Saker, Oscillation of second order nonlinear dynamic equations on time scales, Rocky Mountain J. Math., 34(4), (2004), 1239-1254. MR2095254 (2005e:34113)

[5] M. Bohner and S. H. Saker, Oscillation criteria for perturbed nonlinear dynamic equations, Math. Comput. Modelling, 40(3-4), (2004), 249-260. MR2091058

[6] O. Došlý and S. Hilger, A necessary and sufficient condition for oscillation of the SturmLiouville dynamic equation on time scales, J. Comput. Appl. Math., 141(1-2), (2002), 147158. MR 1908834 (2003f:39015)

[7] L. Erbe, Oscillation criteria for second order linear equations on a time scale, Canad. Appl. Math. Quart., 9 (2001), 1-31. MR1975729 (2004c:39021)

[8] L. Erbe, L. Kong and Q. Kong, Telescoping principle for oscillation for second order differential equations on a time scale, Rocky Mountain J. Math., to appear.

[9] L. Erbe and A. Peterson. Oscillation criteria for second order matrix dynamic equations on a time scale, J. Comput. Appl. Math., 141(1-2), (2002), 169-185. MR1908836(2003e:34023)

[10] L. Erbe and A. Peterson, Boundedness and oscillation for nonlinear dynamic equations on a time scale, Proc. Amer. Math. Soc., 132 (2004), 735-744. MR2019950 (2004i:39015)

[11] L. Erbe and A. Peterson, An oscillation result for a nonlinear dynamic equation on a time scale, Canad. Appl. Math. Quart., to appear.

[12] L. Erbe, A. Peterson, and P. Řehák, Comparison Theorems for Linear Dynamic Equations on Time Scales, J. Math. Anal. Appl., 275 (2002), 418-438. MF1941793 (2003m:39026)

[13] L. Erbe, A. Peterson, and S. H. Saker, Oscillation criteria for second-order nonlinear dynamic equations on time scales, J. London Math. Soc., 67 (2003), 701-714. MR1967701 (2003m:34158)

[14] L. Erbe, A. Peterson, and M. Simon, Square integrability of Gaussian bells on time scales, Comput. Math. Appl., (2005), to appear.

[15] Stefan Hilger, Analysis on measure chains - A unified approach to continuous and discrete calculus, Results Math., 18 (1990), 18-56. MR.1066641 (91m:26027)

[16] Einar Hille, Nonoscillation theorems, Trans. Amer. Math. Soc., 64 (1948), 234-252. MR0027925(10:376c)

[17] B. Kaymakçalan, V. Lakshmikantham and S. Sivasundaram, Dynamic Systems on Measure Chains, Kluwer Academic Publishers, Boston, 1996. MR1419803 (97j:34002)

[18] P. Řehák, Half-linear dynamic equations on time scales: IVP and oscillatory properties, J. Nonlinear Funct. Anal. Appl., 7, (2002), 361-404. MR1946469 (2003h:34067) 
[19] A. Ruffing, J. Lorenz, and K. Ziegler, Difference ladder operators for a harmonic Schrödinger oscillator using unitary linear lattices, J. Comp. Appl. Math., 153 (2003), 395-410. MR $1985710(2004 \mathrm{~h}: 81081)$

[20] Choy-Tak Taam, Nonoscillatory differential equations, Duke Math. J., 19 (1952), 493-497. MR0051994 (14:557d)

[21] A. Wintner, On the comparison theorem of Kneser-Hille, Math. Scand. 5 (1957), 255-260. MR0096867(20:3349)

Department of Mathematics, University of Nebraska-Lincoln, Lincoln, Nebraska $68588-0130$

E-mail address: lerbe@math.unl.edu

Department of Mathematics, University of Nebraska-Lincoln, Lincoln, Nebraska 68588-0130

E-mail address: apeterso@math.unl.edu 